\title{
OPERATOR-SELF-SIMILAR PROCESSES IN A FINITE-DIMENSIONAL SPACE \\ BY
}

\author{
WILLIAM N. HUDSON AND J. DAVID MASON
}

\begin{abstract}
A general representation for an operator-self-similar process is obtained and its class of exponents is characterized. It is shown that such a process is the limit in a certain sense of an operator-normed process and any limit of an operator-normed process is operator-self-similar.
\end{abstract}

1. Introduction. In 1962 Lamperti [8] introduced the notion of a self-similar process, $\{X(t): t \geqslant 0\}$, taking values in a real finite-dimensional inner product space $\widetilde{V}$. A stochastic process $\{X(t)\}$ is called self-similar (s.s.) if it is continuous in probability at each $t \geqslant 0$ and if for every $a>0$ there exist a positive number $B(a)$ and a vector $b(a)$ in $\mathfrak{V}$ such that the process $\{X(a t)\}$ and $\{B(a) X(t)+b(a)\}$ have the same finite-dimensional distributions. Lamperti actually called such processes "semi-stable" but currently this term is being used in another sense. These processes arise in diverse areas (see Taqqu [14]). Self-similar processes are also obtained by various limiting procedures (e.g., see Gorodetskii [4], Kesten and Spitzer [6], Sinai [12], Taqqu [13] and [14], and Weissman [15]).

The definition of "self-similar" was extended by Laha and Rohatgi in [7] to allow scaling by a class of operators on $\mathfrak{V}$. This class was the set of nonsingular positive-definite selfadjoint linear operators on $\mathfrak{V}$. However, the resulting family of processes is not closed under general affine transformations. Properties of positivedefinite selfadjoint linear operators played an important role in their work. In this work we allow scaling by any nonsingular linear operator on $\mathfrak{V}$, but we restrict ourselves to the index set $[0, \infty)$. (Laha and Rohatgi allowed $(0, \infty)$ to be an index set also.)

A stochastic process $\{X(t): t \geqslant 0\}$ is called operator-self-similar (o.s.s.) if it is continuous in law at each $t \geqslant 0$ and if for every $a>0$ there exist a linear operator $B(a)$ on $\mathfrak{V}$ and a vector $b(a)$ in $\mathfrak{V}$ such that the processes $\{X(a t)\}$ and $\{B(a) X(t)+$ $b(a)\}$ have the same finite-dimensional distributions. We say that a process $\{X(t)$ : $t \geqslant 0\}$ is proper if for each $t>0$ the distribution of $X(t)$ is full; that is, the distribution is not contained in a proper hyperplane. All processes considered here are assumed to be proper. Note that for a proper process to be o.s.s., the operators $B(a)$ must be nonsingular.

Received by the editors April 2, 1981.

AMS (MOS) subject classifications (1970). Primary 60G99; Secondary 60F99.

Key words and phrases. Self-similar processes, semistable processes, operator-stable distributions, multivariate stable. 
2. Results and notation. Our first result describes the scaling operators for a proper o.s.s. process. If $D$ is a linear operator on $\mathcal{V}$, then $r^{D}$ will denote the linear operator $\exp ((\ln r) D)=\Sigma_{0}^{\infty}(\ln r)^{j} D^{\prime} / j !$ for $r>0$.

THEOREM 1. Let $\{X(t): t \geqslant 0\}$ be a proper o.s.s. process. Then there exist a linear operator $D$ and a unique continuous function $d:[0, \infty) \rightarrow \mathcal{V}$ such that for each $r>0$, the processes $\{X(r t): t \geqslant 0\}$ and $\left\{r^{D} X(t)+d(r): t \geqslant 0\right\}$ have the same finite-dimensional distributions. Furthermore, if 0 is not an eigenvalue of $D$, then for some $x_{0} \in \mathcal{V}$, $d(r)=\left(I-r^{D}\right) x_{0}$.

Theorem 2 and its corollaries involve certain subsets of the general linear group, $\mathrm{GL}(\mathfrak{V}) .(\mathrm{GL}(\mathfrak{V})$ is the set of all nonsingular linear operators on $\mathfrak{V}$.) For each $t>0$, $G_{t}$ will denote the subset consisting of all operators $A$ in GL( $\left.\mathscr{V}\right)$ such that for some vector $a \in \mathscr{V},\{X(t s)\}$ and $\{A X(s)+a\}$ have the same finite-dimensional distributions. If $\{X(t)\}$ is proper and o.s.s., then for each $r>0, B(r) \in G_{r}$ so $G_{r}$ is nonempty. (To see that $B(r)$ is invertible, note that $X(r) \stackrel{\mathfrak{e}}{=} B(r) X(1)+b(r)$, and use the fact that the distribution of $X(r)$ is full.) Set $G=\cup_{t>0} G_{t}$. The set $G_{1}$ will be called the symmetry group of the process and will also be denoted by $\delta(X(t))$. A linear operator $D$ satisfying Theorem 1 will be referred to as an exponent for the process. In Theorem 2 we describe the set of all exponents for a given process in terms of the tangent space of its symmetry group. The tangent space $T(H)$ of a closed subgroup $H$ of GL( $\mathfrak{V})$ is the set of all linear operators $A$ on $\mathscr{V}$ such that $A=\lim _{n \rightarrow \infty} d_{n}^{-1}\left(D_{n}-I\right)$ for some sequences $\left\{D_{n}\right\}$ in $H$ and $\left\{d_{n}\right\}$ in $(0, \infty)$ where $d_{n} \rightarrow 0$ as $n \rightarrow \infty$, and $I$ is the identity map on $\mathcal{W}$.

Theorem 2. Let $\{X(t): t \geqslant 0\}$ be a proper o.s.s. process and let $\mathcal{E}(X(t))$ be the set of all exponents for $\{X(t)\}$. Then for any $D$ in $\mathcal{E}(X(t))$

$$
\mathcal{E}(X(t))=D+T(\mathcal{S}(X(t))) \text {. }
$$

This result has the following corollaries.

Corollary 1. A proper o.s.s. process has a unique exponent if and only if its symmetry group is finite.

An operator $Q$ on $\mathcal{V}$ is skew-symmetric if $Q+Q^{*}=0$ where $Q^{*}$ is the adjoint of $Q$.

CoRollary 2. If $D$ is an exponent for $\{X(t)\}$, then $\mathcal{E}(X(t))=D+W \mathscr{2}_{0} W^{-1}$ for some subspace $\mathcal{2}_{0}$ of skew-symmetric operators on $\mathfrak{V}$ and some positive-definite selfadjoint linear operator $W$ on $\mathfrak{T}$.

The next theorem characterizes those linear operators $D$ on $\mathcal{V}$ which are exponents for some o.s.s. process. The restrictions on $D$ are a consequence of the requirement that an o.s.s. process be continuous in law at $t=0$. This is illustrated by the following simple example. For $t>0$ set $X(t)=t^{D} X$ where $X$ is any random variable taking values in $\mathcal{V}$ and having a full distribution. Then in order for $\{X(t)\}$ to be o.s.s., it is necessary and sufficient that $t^{D} X$ converges in law to $X(0)$. This condition 
restricts the minimal polynomial of $D$. (Recall that the minimal polynomial of $D$ is the polynomial of smallest degree which annihilates $D$ and its roots are the eigenvalues of $D$.)

TheOREM 3. A linear operator $D$ on $\mathfrak{T}$ is an exponent for some proper o.s.s. process if and only if

(a) every eigenvalue of $D$ has nonnegative real part, and

(b) every eigenvalue of $D$ having real part equal to zero is a simple root of the minimal polynomial of $D$.

The following corollary to Theorem 3 gives the general form for the centering function. This form was obtained in Theorem 1 under the restriction that 0 is not an eigenvalue of the exponent. This restriction can now be removed.

COROLlaRY 3. If $\{X(t)\}$ is proper and o.s.s. with exponent $D$, then for some vector $x$ in $\mathcal{V}, d(r)=\left(I-r^{D}\right) x$. Consequently, the stochastic process $\{X(t)-x\}$ is proper and o.s.s. with exponent $D$ and identically zero centering function.

Our next result connects the initial value $X(0)$ of a proper o.s.s. process with the minimal polynomial of $D$. Let $f=g h$ be the minimal polynomial of $D$ where the roots of $g$ have zero real parts and the real parts of the roots of $h$ are positive. (This factorization is permissible by Theorem 3.) Put $\mathcal{V}_{1}=\operatorname{kernel} g(D)$ and $\mathscr{V}_{2}=$ kernel $h(D)$. Then $\mathfrak{V}=\mathscr{V}_{1} \oplus \mathscr{V}_{2}$. (This follows from the facts that $f(D)=0$ and that $g$ and $h$ are relatively prime. The latter implies that for some polynomials, $p(x)$ and $q(x), p(x) g(x)+q(x) h(x)=1$ and hence $p(D) g(D)+q(D) h(D)=I$.) Clearly $\mathcal{V}_{1}$ and $\mathcal{V}_{2}$ are invariant under $D$ so we may consider the restrictions of $D$ to $\mathcal{V}_{1}$ and to $\mathscr{V}_{2}$.

THEOREM 4. Let $\{X(t): t \geqslant 0\}$ be a proper o.s.s. process which is continuous in probability, and let $D$ be an exponent for $\{X(t)\}$. Then there exist proper o.s.s. processes $\left\{X_{1}(t)\right\}$ and $\left\{X_{2}(t)\right\}$ taking values in $\mathcal{V}_{1}$ and $\mathcal{V}_{2}$ respectively such that

(a) $\left\{X_{1}(t)+X_{2}(t)\right\}$ is a version of $\{X(t)\}$,

(b) $D_{i}=D \mid \mathscr{V}_{i}$ is an exponent for $\left\{X_{i}(t)\right\}, i=1,2$,

(c) $\left\{X_{1}(t)\right\}$ has constant sample paths, and

(d) $X_{2}(0)$ is constant a.s.

The next theorem says that o.s.s. processes arise as limits in certain situations. It is an extension of Theorem 3 of Lamperti [8] to the o.s.s. case.

THEOREM 5. Let $\{X(t): t \geqslant 0\}$ be a proper stochastic process which is continuous in law. If there is a stochastic process $\{Y(t): t \geqslant 0\}$, and if for each $s>0$ there exist $a$ linear operator $A(s)$ on $\mathcal{V}$ and a vector $a(s)$ in $\mathcal{V}$ such that the finite-dimensional distributions of the stochastic process $\{A(s) Y(s t)+a(s): t \geqslant 0\}$ converge to those of $\{X(t)\}$ as $s \rightarrow \infty$, then $\{X(t)\}$ is o.s.s. Conversely, if $\{X(t)\}$ is o.s.s., then $\{X(t)\}$ is such a limit.

Under some mild assumptions it can be shown that the linear operators $A(s)$ in Theorem 5 vary regularly. 
We next consider the case in which the o.s.s. process has as much symmetry as possible. We say that a process $\{X(t)\}$ is elliptically symmetric if $S(X(t))=G_{1}$ is conjugate to the full orthogonal group $\theta$; i.e. $S(X(t))=W \vartheta W^{-1}$ where $W$ is a positive-definite selfadjoint linear operator on $\mathcal{V}$. (If $\mathcal{V}=R^{2}$ and if $S(X(t))=$ $W \circlearrowleft W^{-1}$, then the orbits of nonzero vectors under the action of the group are ellipses.)

THEOREM 6. Let $\{X(t): t \geqslant 0\}$ be a proper o.s.s. process which is continuous in probability. If $\{X(t)\}$ is elliptically symmetric, then $\{X(t)\}$ is s.s. That is, there is a real number $c$ such that $c I$ is an exponent for $\{X(t)\}$.

Our last theorem concerns o.s.s. processes with stationary independent increments. Such processes have operator-stable distributions. In [11], Sharpe defined a probability measure $\mu$ on $\mathcal{V}$ to be operator-stable if there exist sequences of independent identically distributed $\mathcal{T}$-valued random variables $\left\{X_{k}\right\}$, of linear operators $\left\{A_{n}\right\}$ on $\mathcal{T}$, and of vectors $\left\{a_{n}\right\}$ in $\mathcal{V}$ such that $A_{n} \Sigma_{1}^{n} X_{k}+a_{n} \stackrel{\mathfrak{L}}{\rightarrow} \mu$. He proved that full operator-stable laws are infinitely divisible. Thus for all $t>0$, the powers, $\hat{\mu}(y)^{t}$, of the characteristic function, $\hat{\mu}(y)$, of $\mu$ are characteristic functions of infinitely divisible distributions $\mu^{t}$. He showed that if $Y(t)$ is a $\mathcal{V}$-valued random variable with distribution $\mu^{t}$, then there exist a nonsingular linear operator $B$ on $\mathcal{V}$ and a vector $b(t)$ in $\mathcal{T}$ such that $Y(t) \stackrel{\mathcal{E}}{=} t^{B} X(1)+b(t)$. We will use Sharpe's notation below; that is, if $A$ is a linear operator on $\mathcal{V}$ and $Y$ is a $\mathcal{V}$-valued random variable with distribution $\mu$, then $A Y$ has distribution $A \mu=\mu A^{-1}$. Thus Sharpe's theorem says that $\mu^{t}=t^{B} \mu * \delta(b(t))$ where $*$ denotes convolution and $\delta(b(t))$ is the probability measure on $\mathcal{T}$ assigning mass one to $b(t)$. Following Sharpe, we refer to $B$ as an (operator-stable) exponent for $\mu$. (However, $B^{-1}$ would be the usual exponent for a one-dimensional stable law.)

THEOREM 7. Let $\{X(t): t \geqslant 0\}$ be a proper stochastic process which is continuous in probability and has stationary independent increments with $X(0)=0$. Then $\{X(t)\}$ is $o . s . s$. if and only if $X(1)$ has a full operator-stable distribution with centering function identically equal to zero. In this case the o.s.s. exponent is the same as the operator-stable exponent.

There are certain remarks that should be made. First, there is an association between a proper operator-self-similar process and a stationary process. If $\{X(t)$ : $t \geqslant 0\}$ is proper and o.s.s. with an exponent $D$ and centering term $d(t) \equiv 0$, and if $Y(t)=e^{-t D} X\left(e^{t}\right),-\infty<t<\infty$, then $\{Y(t)\}$ is a stationary process. Conversely, let $\{Y(t):-\infty<t<\infty\}$ be a proper stationary process and let $D$ be a nonsingular linear operator on $\mathcal{V}$ such that $t^{D} Y(\ln t)$ converges in law to, say, $\nu$ as $t \rightarrow 0$. For $t>0$, define $X(t)$ to be $t^{D} Y(\ln t)$, and for $t=0$, let $X(0)$ be a random variable with distribution $\nu$. Then $\{X(t): t \geqslant 0\}$ is proper and o.s.s. with exponent $D$ and centering function $d(t) \equiv 0$. These statements are easy to establish.

Next suppose that $\{X(t): t \geqslant 0\}$ is proper, o.s.s., and Gaussian. Let $\mathfrak{V}=R^{N}$ and let $\rho(s, t)$ be the covariance operator of $X(s)$ and $X(t)$. Assume $E X(t) \equiv 0$. When $N=1$ and $\alpha>0$ is an exponent for $\{X(t)\}$, Lamperti [8] showed that for positive 
numbers $r, s, t, \rho(r s, r t)=r^{2 \alpha} \rho(s, t)$. In fact, he showed that this condition is necessary and sufficient for a Gaussian process to be s.s. This can be extended to the o.s.s. case. If the linear operator $D$ is an exponent for a proper Gaussian o.s.s. process $\{X(t): t \geqslant 0\}$ with mean function identically equal to zero, then for all positive numbers, $r, s$, and $t$,

$$
\rho(r s, r t)=r^{D} \rho(s, t) r^{D^{*}}
$$

Conversely if a mean zero Gaussian process has a covariance operator satisfying the above equality for some linear operator $D$ on $R^{N}$, then it is o.s.s. with exponent $D$. This, too, is easy to establish.

It is possible to modify some of the above theorems so that they are concerned only with the $k$-dimensional distributions of the process $\{X(t)\}$. One might define a stochastic process $\{X(t): t \geqslant 0\}$ taking values in $\mathcal{V}$ to be $k$-operator-self-similar if $\{X(t)\}$ is continuous in law and if for any $r>0$ there exist a linear operator $B(r)$ on $\mathcal{V}$ and a vector $b(r)$ in $\mathcal{V}$ such that for any positive numbers $t_{1}, \ldots, t_{k}$

$$
\left(X\left(r t_{1}\right), \ldots, X\left(r t_{k}\right)\right) \stackrel{\mathfrak{e}}{=}\left(b(r) X\left(t_{1}\right)+b(r), \ldots, B(r) X\left(t_{k}\right)+b(r)\right) .
$$

If, for example in Theorem 1, one only assumes that $\{X(t): t \geqslant 0\}$ is $k$-o.s.s., then the process still has an exponent. That is, there is a linear operator $D$ on $\mathcal{V}$ such that $B(r)$ may be chosen to be $r^{D}$. Analogues exist for the other theorems.

Finally, it is easy to see that in the definition of an o.s.s. process it is only necessary to assume continuity at a single nonzero point.

We now state as propositions three facts used several times in the sequel.

Proposition 1. A probability measure $\mu$ on $\mathfrak{V}$ is not full (i.e. is concentrated in a proper hyperplane) if and only if there exists a nonzero vector $y$ in $\mathcal{V}$ such that for all real numbers $c,|\hat{\mu}(c y)|=1$.

This is Proposition 1 of Sharpe [11].

Proposition 2. Let $\mathcal{H}$ be a compact subgroup of $\mathrm{GL}(\mathfrak{V})$. Then there exist a positive-definite selfadjoint linear operator $W$ and a closed subgroup $\vartheta_{0}$ of the orthogonal group $\vartheta$ such that $\mathcal{H}=W \vartheta_{0} W^{-1}$.

This is a classical result and is contained in Theorem 5 of Billingsley [1].

PROPOSITION 3. The tangent space of the orthogonal group 0 is the vector-space 2 of skew-symmetric linear operators.

To see this, note that $Q \in T(\theta)$ implies that $\exp (s Q) \exp \left(s Q^{*}\right)=I$ for all $s$, so by differentiation and evaluation at $s=0$ we see that $Q$ is skew-symmetric. For the converse, since any $Q \in \mathcal{2}$ commutes with $Q^{*}$, we have $\exp (s Q) \exp \left(s Q^{*}\right)=I$ for all $s$, i.e. $Q \in T(\theta)$.

3. Proof of Theorem 1. Since many of our lemmas concern equality of the finite-dimensional distributions of two processes, we use the following notation.

If $T=\left(t_{1}, \ldots, t_{k}\right) \in[0, \infty)^{k}$ and $r \in R^{1}$, then $r T=\left(r t_{1}, \ldots, r t_{k}\right)$ and $X(T)=$ $\left(X\left(t_{1}\right), \ldots, X\left(t_{k}\right)\right)$. Also, for $A$ a linear operator on $\mathcal{V}, A X(T)=\left(A X\left(t_{1}\right), \ldots, A X\left(t_{k}\right)\right)$, 
and for $c \in \mathcal{V}, \underline{c}=(c, \ldots, c) \in \mathcal{V}^{k}$. Other notation is as in $\S 2$. Throughout this section we assume that $\{X(t): t \geqslant 0\}$ is a proper o.s.s.

LEMMA 3.1. If there are two distinct positive numbers $r$ and s such that $G_{r} \cap G_{s} \neq \varnothing$, then

(a) there is a vector $c \in \mathcal{V}$ such that $\{X(r t)\}$ and $\{X(s t)+c\}$ have the same finite-dimensional distributions, and

(b) $G_{r}=G_{s}$.

Proof. Since (b) is immediate from (a) and the definition of the $G_{t}$ 's, we need only prove (a). Let $A \in G_{r} \cap G_{s}$. Then there are vectors $a_{r}$ and $a_{s}$ in $\mathcal{V}$ such that $X(r T) \stackrel{\mathfrak{E}}{=} A X(T)+\underline{a}_{r}$ and $X(s T) \stackrel{\mathfrak{E}}{=} A X(T)+\underline{a}_{s}$. Hence, $X(r T) \stackrel{\mathfrak{e}}{=} X(s T)+\underline{c}$ where $c=a_{r}-a_{s}$. Q.E.D.

LemMA 3.2. (a) If $A_{n} \in G_{r_{n}}$ with $r_{n} \rightarrow 0$ and if $A_{n} \rightarrow A \in \mathrm{GL}(\mathcal{V})$, then $X(T) \stackrel{\mathfrak{e}}{=} X(\underline{0})$ for all $T$.

(b) If for some pair of distinct positive numbers $r$ and $s, G_{r}=G_{s}$, then $X(T) \stackrel{\mathfrak{E}}{=} X(\underline{0})$ for all $T$.

Proof. Since $A_{n} \in G_{r_{n}}$, there are $a_{n} \in \mathfrak{V}$ such that $X\left(r_{n} T\right) \stackrel{\mathfrak{e}}{=} A_{n} X(T)+\underline{a}_{n}$. Since $X(t)$ is continuous in law and $A_{n} \rightarrow A \in \mathrm{GL}(\mathcal{V})$, there is $a \in \mathcal{V}$ such that $a_{n} \rightarrow a$. Hence, by continuity in law, $X(0) \stackrel{\mathcal{e}}{=} A X(T)+\underline{a}$. Therefore, for all $T$,

$$
X(T) \stackrel{\mathfrak{e}}{=} A^{-1}(X(\underline{0})-\underline{a}) .
$$

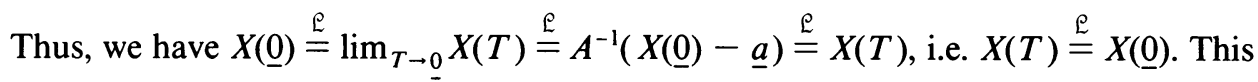
proves (a).

Now assume $G_{r}=G_{s}$ with $r<s$. We start by showing that for any $t>0$, $G_{t r}=G_{t s}$. It follows from Lemma 3.1 that there is a vector $c \in \mathcal{V}$ such that for all $T=\left(t_{1}, \ldots, t_{k}\right), X(r T) \stackrel{e}{=} X(s T)+\underline{c}$. Replacing $T$ by $t T$ we obtain for all $T$, $X(t r T) \stackrel{\mathscr{e}}{=} X(t s T)+\underline{c}$. This implies $G_{t r}=G_{t s}$. Now replace $t$ by $1 / s$ to obtain $G_{r / s}=$ $G_{1}$. Since $r / s<1$, the above argument implies that for any $t>0, G_{t r / s}=G_{t}$. So, if $t=r^{n} / s^{n}$, we obtain $G_{\left(r^{n+1} / s^{n+1}\right)}=G_{\left(r^{n} / s^{n}\right)}$ for $n=1,2, \ldots$, and these equalities combine to yield $G_{\left(r^{n} / s^{n}\right)}=G_{1}, n=1,2, \ldots$ Note that $r^{n} / s^{n} \rightarrow 0$. Since $I$ is obviously in $G_{1}$, put $A_{n}=I$ and use part (a) to finish the proof. Q.E.D.

LEMMA 3.3 The set $G=\cup_{t>0} G_{t}$ is a subgroup of $\mathrm{GL}(\mathfrak{V})$. In particular,

(a) $I \in G_{1}$,

(b) $A \in G_{r}$ implies $A^{-1} \in G_{1 / r}$, and

(c) $A \in G_{r}$ and $B \in G_{s}$ imply $A B \in G_{r s}$.

Proof. Conclusion (a) is obvious.

In order to prove (b), let $A \in G_{r}$. Then there is $a \in \mathcal{V}$ such that for all $T, X(r T)$ $\stackrel{\mathfrak{e}}{=} A X(T)+\underline{a}$. Replace $T$ by $(1 / r) T$ and solve for $X((1 / r) T)$ to obtain $X((1 / r) T) \stackrel{\mathfrak{e}}{=} A^{-1} X(T)-A^{-1} \underline{a}$ which implies $A^{-1} \in G_{1 / r}$ and proves (b). 
For (c), let $A \in G_{r}$ and $B \in G_{s}$. Then $X(r T) \stackrel{\stackrel{\mathfrak{e}}{=}}{A} X(T)+\underline{a}$ and $X(s T) \stackrel{\stackrel{\mathfrak{e}}{=}}{B} X(T)$ $+\underline{b}$ for some $a, b \in \mathfrak{V}$. In the first equality, replace $T$ by $s T$ and then use the second equality to obtain $X(r s T) \stackrel{\stackrel{\mathcal{E}}{=}}{A B X}(T)+A \underline{b}+\underline{a}$ which implies $A B \in G_{r s}$ and proves (c). Q.E.D.

Lemma 3.4. The subgroup $G$ is closed in $\mathrm{GL}(\mathscr{V})$. In particular, if $A_{n} \in G_{r_{n}}$ and $A_{n} \rightarrow A \in \mathrm{GL}(\mathbb{V})$, then

(a) $A \in G_{1}$ and $G=G_{1}$,or

(b) there is a number $r>0$ such that $r_{n} \rightarrow r$ and $A \in G_{r}$. (These two conclusions are not mutually exclusive.)

Proof. We first consider the case when $\liminf r_{n}=0$. Then by Lemma 3.2(a), $X(T) \stackrel{\mathfrak{e}}{=} X(\underline{0})$. It follows immediately that for all $s>0, G_{s}=G_{1}$ and hence $G=G_{1}$. Let $\left\{r_{n^{\prime}}\right\}$ be a subsequence of $\left\{r_{n}\right\}$ which converges to 0 . Then for some $a_{n^{\prime}} \in \mathscr{V}$,

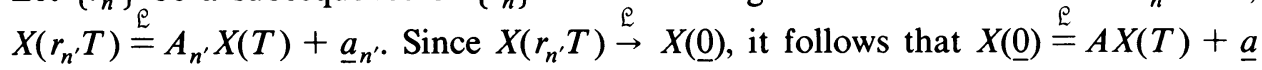
for some $a \in \mathfrak{V}$. Hence, $X(T) \stackrel{\stackrel{\mathfrak{e}}{=}}{A} X(T)+\underline{a}$ which implies $A \in G_{1}$. Now consider the case $\lim \sup r_{n}=\infty$. Then $A_{n}^{-1} \in G_{1 / r_{n}}, A_{n}^{-1} \rightarrow A^{-1}$ and $\liminf 1 / r_{n}=0$, so the result follows from the previous case. Finally, when $0<\liminf r_{n} \leqslant \lim \sup r_{n}<\infty$, let $\left\{r_{n^{\prime}}\right\}$ denote an arbitrary convergent subsequence of $\left\{r_{n}\right\}$ with limit $t$. Then again for some $a_{n^{\prime}} \in \mathfrak{V}, X\left(r_{n^{\prime}} T\right) \stackrel{\stackrel{\mathcal{E}}{=}}{=} A_{n^{\prime}} X(T)+\underline{a}_{n^{\prime}}$. Hence, $X(t T) \stackrel{\mathcal{E}}{=} A X(T)+\underline{a}$ for some $a \in \mathfrak{V}$ and so $A \in G_{t}$. Now if $t_{*}=\liminf r_{n_{\rho}}<\lim \sup r_{n}=t^{*}$, we see that $A \in G_{t^{*}}$ $\cap G_{t^{*}}$ and so by Lemmas 3.1 and 3.2, $X(T) \stackrel{\mathcal{e}}{=} X(\underline{0})$. Again, this implies that $G=G_{1}$ and (a) holds. If $t_{*}=t^{*}$, then $r_{n} \rightarrow t$ and we have proved (b). Q.E.D.

Definition. When $G \neq G_{1}$, define $\eta: G \rightarrow R^{1}$ by $\eta(A)=\ln s$ whenever $A \in G_{s}$.

LemmA 3.5. When $G \neq G_{1}$, the function $\eta$ is a continuous homomorphism between the group $G$ and the additive group $R^{1}$.

Proof. The lemma follows from Lemmas 3.3 and 3.4 once we show that $\eta$ is well defined. To do this, it suffices to show that if $r$ and $s$ are distinct positive numbers, then $G_{r} \cap G_{s}=\varnothing$. If this is not so, then $X(T) \stackrel{\stackrel{\mathfrak{e}}{=}}{(\underline{0}}(\underline{)})$ and hence $G=G_{1}$ contrary to hypothesis. Therefore, $\eta$ is well defined. Q.E.D.

Lemma 3.6. If $G \neq G_{1}$, then $G_{1}$ is not a neighborhood of $I$ in $G$.

Proof. Let $r_{n}=1+1 / n, n=1,2,3, \ldots$ We will show that there exists a sequence $\left\{\bar{A}_{n}\right\}$ in $G \backslash G_{1}$ which converges to $I$. Since $G_{r_{n_{\mathfrak{e}}}} \neq \varnothing$, select $A_{n} \in G_{r_{n}}$ and $a_{n} \in \mathfrak{V}$ such that $X\left(r_{n} T\right) \stackrel{\mathfrak{E}}{=} A_{n} X(T)+\underline{a}_{n}$. Now $X\left(r_{n} T\right) \stackrel{r_{n_{\mathfrak{L}}}}{\rightarrow} X(T)$, so by the convergence of types theorem (see Sharpe [11] or Billingsley [1]) $\left\{A_{n}\right\}$ is precompact in $\mathrm{GL}(\mathfrak{V})$. Hence, there exists a subsequence $\left\{A_{n^{\prime}}\right\}$ of $\left\{A_{n}\right\}$ which converges to some $A \in \mathrm{GL}(\mathcal{V})$. By Lemma 3.4, $A \in G_{1}$. Set $\overline{A_{n}}=A_{n^{\prime}} A^{-1}$. Then $\overline{A_{n}} \rightarrow I$ and $\overline{A_{n}} \in G_{r_{n}}$ by Lemma 3.3. It remains to show that for all $n, \overline{A_{n}} \notin G_{1}$. But, $\bar{A}_{n} \in G_{1}$ implies $G_{r_{n}} \cap G_{1} \neq \varnothing$ and hence $G=G_{1}$ contrary to hypothesis. Q.E.D. 
Now we prove Theorem 1. Assume $G=G_{1}$. Then by Lemmas 3.1 and 3.2, $X(r T) \stackrel{\mathfrak{e}}{=} X(T)$ for all $r>0$ and for all $T=\left(t_{1}, \ldots, t_{k}\right)$. Thus in this case we may take $D=0$ and $d(r) \equiv 0$.

Now assume $G \neq G_{1}$. Let $T(G)$ denote the tangent space to $G$ at the identity $I$. For $A \in T(G)$, the exponential $e^{A}$ of $A$ is defined by $e^{A}=\sum_{0}^{\infty}(j !)^{-1} A^{j}$. It is well known (see, e.g. Cohn [2]) that the image of $T(G)$ under the exponential map is a neighborhood of the identity in $G$. Thus by Lemma 3.6, the image is not a subset of $G_{1}$ and so there exists an operator $A \in T(G)$ such that $e^{A} \notin G_{1}$. Consider the map $s \rightarrow \eta\left(e^{s A}\right)$. It is a continuous homomorphism from $\left(R^{1},+\right)$ into $\left(R^{1},+\right)$ and hence $\eta\left(e^{s A}\right)=\alpha s$ for some $\alpha \in R^{1}$. If $\alpha=0, e^{s A} \in G_{1}$ for $s=1$ contrary to the choice of $A$. Thus $\alpha \neq 0$. Set $D=(1 / \alpha) A$. Then for all $s, \eta\left(e^{s D}\right)=s$, i.e. $\eta\left(r^{D}\right)=\ln r$ for all $r>0$. This implies that $r^{D} \in G_{r}$ for every $r>0$. Hence, there exists $d(r) \in \mathcal{V}$ such that $X(r t) \stackrel{\mathfrak{e}}{=} r^{D} X(T)+d(r)$. Therefore the processes $\{X(r t): t \geqslant 0\}$ and $\left\{r^{D} X(t)\right.$ $+d(r): t \geqslant 0\}$ have the same finite-dimensional distributions. From the equality $X(r t) \stackrel{\mathfrak{e}}{=} r^{D} X(t)+d(r)$ and the continuity in law, we see that the function $d$ is unique and continuous.

Finally, we show that $d(r)=\left(I-r^{D}\right) x_{0}$ for some $x_{0} \in \mathcal{V}$ whenever 0 is not a eigenvalue of $D$. For all $s, r, t$ positve numbers,

$$
(s r)^{D} X(t)+d(s r) \stackrel{\mathfrak{e}}{=} X(s r t) \stackrel{\mathfrak{e}}{=} s^{D} X(r t)+d(s) \stackrel{\mathfrak{e}}{=} s^{D} r^{D} X(t)+s^{D} d(r)+d(s) .
$$

Hence, $d(s r)=s^{D} d(r)+d(s)$. By symmetry, $d(s r)=r^{D} d(s)+d(r)$. Therefore, $s^{D} d(r)+d(s)=r^{D} d(s)+d(r)$, i.e. $\left(I-r^{D}\right) d(s)=\left(I-s^{D}\right) d(r)$. For $r$ and $s$ such that $\left(I-r^{D}\right)$ and $\left(I-s^{D}\right)$ are nonsingular, we have $\left(I-r^{D}\right)^{-1} d(r)=$ $\left(I-s^{D}\right)^{-1} d(s)$, i.e. $\left(I-r^{D}\right)^{-1} d(r)=x_{0}$ for some $x_{0} \in \mathcal{V}$, for all $r \in \mathbb{Q}$ where $\mathcal{Q}$ is a dense subset of $[0, \infty)$. Hence, $d(r)=\left(I-r^{D}\right) x_{0}$ for all $r \in \mathbb{Q}$. Since $d$ is continuous, $d(r)=\left(I-r^{D}\right) x_{0}$ for all $r>0$. Q.E.D.

4. Proof of Theorem 2 and its corollaries. The tangent space of a closed subgroup $H$ of GL( $\mathfrak{V})$ is the set of all linear operators $A$ on $\mathfrak{V}$ such that $A=\lim d_{n}^{-1}\left(D_{n}-I\right)$ for some sequence $\left\{D_{n}\right\}$ in $H$ and $0<d_{n} \rightarrow 0$.

Throughout this section $\{X(t)\}$ is assumed to be a proper o.s.s. process.

LEMMA 4.1. $\mathcal{E}(X(t)) \subset T(G)$.

Proof. For $D \in \mathcal{E}(X(t)), t^{D} \in G_{t}$ for all $t>0$. Hence, $e^{t D} \in G$ for all $t \in R^{1}$. Since $D=\lim _{t \rightarrow 0} t^{-1}\left(e^{t D}-I\right), D \in T(G)$. Q.E.D.

LeMma 4.2. If $G=G_{1}$, then $\mathcal{E}(X(t))=T(G)$.

Proof. Let $D \in T(G)$. Since $T(G)$ is a vector space, $(\ln r) D \in T(G)$ for all $r>0$. Hence, $r^{D} \in G=G_{1}$ for $r>0$. Therefore for some $d(r) \in \mathcal{V}, X(r) \stackrel{\mathscr{E}}{=} r^{D} X(1)+d(r)$. But $G=G_{1}$ implies $X(T) \stackrel{\mathfrak{e}}{=} X(\underline{0})$. Thus $X(r t) \stackrel{\mathfrak{e}}{=} r^{D} X(t)+d(r)$, so $D \in \mathcal{E}(X(t))$. Q.E.D.

Since $G=G_{1}$ implies that $0 \in \mathcal{E}(X(t))$, this lemma proves Theorem 2 in this case. In $\S 3$ the function $\eta: G \rightarrow R^{1}$ was defined when $G \neq G_{1}$ and it was showed that $\eta$ is 
a continuous homomorphism between the group $G$ and the additive group $R^{1}$. Now for $G \neq G_{1}$ define $L: T(G) \rightarrow R^{1}$ by $L(A)=\eta\left(e^{A}\right)$.

Lemma 4.3. The function $L: T(G) \rightarrow R^{1}$ defined whenever $G \neq G_{1}$ is a linear functional.

Proof. We first show that for $A \in T(G)$ and $t \in R^{1}, L(t A)=t L(A)$. We showed in the proof of Theorem 1 that for $A$ fixed, the map $s \rightarrow \eta\left(e^{s A}\right)$ is a continuous homomorphism, so there is an $\alpha \in R^{1}$ such that $\eta\left(e^{s A}\right)=\alpha s$ for all $s$. Then $L(t A)=t \alpha=t L(A)$.

Next, we show that for $A, B \in T(G), L(A+B)=L(A)+L(B)$. It is well known (e.g., Cohn [2, p. 111]) that as $t \rightarrow 0, e^{t A} e^{t B}=e^{t(A+B)+O\left(t^{2}\right)}$. Thus, if $Z(A, B)$ $\equiv E^{-1}\left(e^{A} e^{B}\right)$, where $E^{-1}$ denotes the inverse of the exponential map which is defined in some neighborhood of $I$ in $G, \lim _{t \rightarrow 0} t^{-1} Z(t A, t B)=A+B$. Hence,

$$
\begin{aligned}
L(A+B) & =L\left(\lim _{t \rightarrow 0} t^{-1} Z(t A, t B)\right)=\lim _{t \rightarrow 0} t^{-1} L(Z(t A, t B)) \\
& =\lim _{t \rightarrow 0} t^{-1}(L(t A)+L(t B))=L(A)+L(B) . \quad \text { Q.E.D. }
\end{aligned}
$$

LEMMA 4.4. When $G \neq G_{1}, \operatorname{ker} L=T\left(G_{1}\right)$.

Proof. For $A \in T\left(G_{1}\right)$, $e^{A} \in G_{1}$, so $L(A)=0$. Conversely, for $A$ such that $L(A)=0, L(t A)=0$ for all $t \in R^{1}$. Hence $e^{t A} \in G_{1}$ for all $t \in R^{1}$. Thus $A \in T\left(G_{1}\right)$ since $A=\lim _{t \rightarrow 0} t^{-1}\left(e^{t A}-I\right)$. Q.E.D.

LEMMA 4.5. If $G \neq G_{1}$, then

$$
\mathcal{E}(X(t))=\{A \in T(G): L(A)=1\} .
$$

Proof. Since $A \in \mathcal{E}(X(t))$ if and only if $t^{A} \in G_{t}$ for all $t>0$, we have $e^{A} \in G_{e}$, so $L(A)=1$. Conversely, for $A \in T(G)$ with $L(A)=1, L((\ln t) A)=\ln t$ for $t>0$. Hence, $t^{A} \in G_{t}$ for all $t>0$, so $A \in \mathcal{E}(X(t))$. Q.E.D.

Lemma 4.6. When $G \neq G_{1}$, if $D_{1}$ and $D_{2}$ are in $\mathcal{E}(X(t))$, then $D_{1}-D_{2} \in T\left(G_{1}\right)$.

Proof. Since $D_{i} \in \mathcal{E}(X(t)), i=1,2, L\left(D_{1}\right)=L\left(D_{2}\right)$. Hence $L\left(D_{1}-D_{2}\right)=0$, so $D_{1}-D_{2} \in \operatorname{ker} L$. But $\operatorname{ker} L=T\left(G_{1}\right)$. Q.E.D.

Lemma 4.7. When $G \neq G_{1}$, if $D \in \mathcal{E}(X(t))$ and $A \in T\left(G_{1}\right)$, then $D+A \in \mathcal{E}(X(t))$.

Proof. We have $L(D)=1$ and $L(A)=0$, so $L(t(A+D))=t$ for all $t \in R^{1}$. As before, this implies $A+D \in \mathcal{E}(X(t))$. Q.E.D.

Now to complete the proof of Theorem 2, note that $G_{1}=\delta(X(t))$ and let $D$ be an exponent for $\{X(t)\}$. Lemma 4.6 implies that $\delta(X(t)) \subset D+T(\delta(X(t)))$, while Lemma 4.7 yields the reverse inclusion. This proves Theorem 2.

For the proof of Corollary 1 , note that from Theorem 2 we have $\{X(t)\}$ has a unique exponent if and only if $T(\delta(X(t)))=\{0\}$. Now the image of the tangent space of a closed subgroup of GL(V) under the exponential map contains a neighborhood of the identity. Also the tangent space is a vector space and the exponential map is continuous. Hence $\{I\}$ is open in $\delta(X(t))$ if and only if $T(\delta(X(t)))=\{0\}$. Since $\delta(X(t))$ is always compact, $\{I\}$ is open in $\delta(X(t))$ if and only if $\delta(X(t))$ is a finite set. 
Finally, we prove Corollary 2. We have $\mathcal{E}(X(t))=D+T(\delta(X(t)))$ for any $D \in \mathcal{E}(X(t))$. Since the group $\delta(X(t))$ is compact, there is a positive-definite selfadjoint linear operator $W$ on $\mathcal{V}$ such that $\delta(X(t))=W \vartheta_{0} W^{-1}$ where $\theta_{0}$ is a closed subgroup of the full orthogonal subgroup of GL(V) $)$. Then $\delta\left(W^{-1} X(t)\right)=\theta_{0}$. By Proposition 3, $T\left(\vartheta_{0}\right)=\mathscr{2}_{0}$ where $\mathscr{Q}_{0}$ is a subspace of skew-symmetric linear operators on $\mathcal{V}$. Hence $T\left(\delta(X(t))=W_{2_{0}} W^{-1}\right.$. Q.E.D.

5. Proof of Theorem 3. Let $D$ be an exponent for a proper o.s.s. process $\{X(t)\}$. First, we show that $D$ satisfies conditions (a) and (b).

Let $\mu_{t}$ denote the distribution of $X(t)$. We may assume that the distributions $\mu_{t}$ have been symmetrized if necessary to ensure that for all $s>0$ and all $t>0$

$$
\mu_{s t}=t^{D} \mu_{s} \text {. }
$$

Suppose (a) is false. Then $D$, and hence its adjoint $D^{*}$, have an eigenvalue with negative real part. This implies that there is a nonzero vector $x$ in $\mathcal{V}$ such that $\lim _{t \rightarrow \infty} t^{D^{*}} x=0$. Put $s=1 / t$ in 5.1 to obtain the equation

$$
\mu_{1}=t^{D} \mu_{1 / t}, \quad t>0 .
$$

Then for any real number $c$, the characteristic functions of $\mu_{1}$ and $\mu_{1 / t}$ satisfy the equality

$$
\hat{\mu}_{1}(c x)=\hat{\mu}_{1 / t}\left(c t^{D^{*}} x\right)
$$

Since $\{X(t)\}$ is continuous in law $\hat{\mu}_{1 / t} \rightarrow \hat{\mu}_{0}$ as $t \rightarrow \infty$ and hence by 5.3

$$
\hat{\mu}_{1}(c x)=\hat{\mu}_{0}(0)=1 \text {. }
$$

But according to Proposition 1, (5.4) implies that $\mu_{1}$ is not full. This contradicts the hypothesis that $\{X(t)\}$ is proper. This proves (a).

Now let $f(x)=g(x) h(x)$ be the minimal polyinomial of $D$ and $D^{*}$ where $g(x)$ and $h(x)$ are polynomials, the roots of $g(x)$ have real parts equal to zero, and the roots of $h(x)$ have positive real parts. Put $\mathcal{G}=$ kernel of $g\left(D^{*}\right)$ and $\mathcal{H}=$ kernel of $h\left(D^{*}\right)$. Then $\mathcal{V}=\mathcal{G} \oplus \mathcal{H}$ and both $\mathcal{G}$ and $\mathcal{H}$ are invariant under $D^{*}$. The minimal polynomial of the restriction of $D^{*}$ to $\mathcal{G}$ is $g(x) ; g(x)$ has a factorization of the form $g(x)=x^{m_{0}}\left(x^{2}+\beta_{1}^{2}\right)^{m_{1}} \cdots\left(x^{2}+\beta_{n}^{2}\right)^{m_{n}}$ where $\beta_{1}, \ldots, \beta_{n}$ are distinct positive numbers, $m_{1}, \ldots, m_{n}$ are positive integers and $m_{0}$ is a nonnegative integer. In order to prove (b), we have to show that $m_{i} \leqslant 1$ for all $i$. For $j \geqslant 1$ put

$$
w_{j}=\operatorname{kernel}\left(\left(D^{*}\right)^{2}+\beta_{j}^{2}\right)^{m_{j}}
$$

and for $j=0$ put $\mathscr{W}_{0}=\operatorname{kernel}\left(D^{*}\right)^{m_{0}}$; then $\mathcal{G}=\mathscr{W}_{0} \oplus \cdots \oplus \mathcal{W} \int_{n}$ and each $\mathcal{W}_{j}$ is invariant under $D^{*}$. Let $D_{j}^{*}=D^{*} \mid \mho_{j}$. Fix $j$ in $\{0, \ldots, n\}$ and set $D_{j}^{*}=S^{*}+N^{*}$ where $S^{*}$ is semisimple, $N^{*}$ is nilpotent and $S^{*}$ and $N^{*}$ commute (see [5, Theorem 13, p. 267]). In order to show that $m_{j} \leqslant 1$, it suffices to show that $N^{*}=0$ [5, Theorem 11, p. 264]. We know that the minimal polynomial of $S^{*}$ is $x^{2}+\beta_{j}^{2}$ for $j \geqslant 1$ or $x$ if $j=0$ (see the proof of Theorem 13 on p. 268 of [5]). This fact together with the Cyclic Decomposition Theorem [5, p. 233] can be used to show that for 
$j \geqslant 1 S^{*}$ has a matrix representation of the form $S^{*}=\operatorname{diag} .(B, \ldots, B)$ where

$$
B=\left(\begin{array}{cc}
0 & -\beta_{j} \\
\beta_{j} & 0
\end{array}\right)
$$

with respect to some basis. The corresponding representation for $t^{S^{*}}$ is diag. $\left(t^{B}, \ldots, t^{B}\right)$ and the power series expansion can be used to obtain

$$
t^{B}=\left(\begin{array}{cc}
\cos \left(\beta_{j} \ln t\right) & -\sin \left(\beta_{j} \ln t\right) \\
\sin \left(\beta_{j} \ln t\right) & \cos \left(\beta_{j} \ln t\right)
\end{array}\right) .
$$

It is clear from this that we can select a sequence of positive numbers $t_{n}$ converging to zero such that $t_{n}^{S^{*}}=I$, the identity on $\mho_{j}$. When $j=0, S^{*}=0$ so $T^{S^{*}}=I$ for all $t$. Now suppose $N^{*} \neq 0$. Let $k>0$ be such that $t^{N^{*} y}=\sum_{i=0}^{k}(\ln t)^{i}(i !)^{-1}\left(N^{*}\right)^{i} y$ where $\left(N^{*}\right)^{k} y \neq 0$ for some fixed $y$ in $\mathcal{T}$. Define $\alpha(t)=(k !) /(\ln t)^{k}$. Then for any $c \in R$ (setting $s=1$ in (5.1)),

$$
\hat{\mu}_{t_{n}}\left(\alpha\left(t_{n}\right) c y\right)=\hat{\mu}_{1}\left(\alpha\left(t_{n}\right) c t_{n}^{D^{*}} y\right) .
$$

Now as $n \rightarrow \infty, \alpha\left(t_{n}\right) \rightarrow 0$ and so

$$
\lim _{n \rightarrow \infty} \hat{\mu}_{t_{n}}\left(\alpha\left(t_{n}\right) c y\right)=\hat{\mu}_{0}(0)=1 \text {. }
$$

On the other hand, $t_{n}^{D^{*}} y=t_{n}^{N^{*}} t_{n}^{S^{*}} y=t_{n}^{N^{*}} y$ so $\lim _{n \rightarrow \infty} c \alpha\left(t_{n}\right) t_{n}^{D^{*}} y=c\left(N^{*}\right)^{k} y$. Thus for every real number $c, \hat{\mu}_{1}\left(c\left(N^{*}\right)^{k} y\right)=1$ which again implies that $\mu_{1}$ is not full and we obtain the same contradiction as before. This proves (b).

We now prove the converse. Let $D$ be any linear operator on $\mathcal{V}$ which satisfies conditions (a) and (b) of Theorem 3. As in the first part of the proof let $f=g h$ be the minimal polynomial of $D$ and $D^{*}$ where the roots of $g$ have zero real parts and those of $h$ have positive real parts. Put $\mathscr{V}_{1}=$ kernel $g(D)$ and $\mathcal{V}_{2}=$ kernel $h(D)$ so that $\mathcal{V}=\mathcal{V}_{1} \oplus \mathcal{V}_{2}$. Let $D_{1}$ be the restriction of $D$ to $\mathcal{V}_{1}$. The minimal polynomial of $D_{1}$ is $g$ and every root of $g$ is simple so $D_{1}$ is semisimple. Since every eigenvalue of $D_{1}$ has real part zero, $\left\{t^{D_{1}}: t>0\right\}^{-}$is a compact group of linear operators on $\mathcal{V}_{1}$. Now let $X_{1}$ and $X_{2}$ be independent random vectors which are full on and take values in $\mathcal{V}_{1}$ and $\mathcal{V}_{2}$ respectively and such that the distribution of $X_{1}$ is invariant under the group $\left\{t^{D_{1}}: t>0\right\}^{-}$. For $t>0$, define $X(t)=t^{D}\left(X_{1}+X_{2}\right)$, and for $t=0$ set $X(0)=X_{1}$. To see that $X(t)$ is continuous in law we need only check that $X(t) \stackrel{\mathfrak{L}}{\rightarrow} X_{1}$ as $t \rightarrow 0$. But $t^{D} X_{2} \rightarrow 0$ since the eigenvalues of $D \mid \mathscr{V}_{2}$ are positive. Since $t^{D} X_{1} \stackrel{e}{=} X_{1}$ for all $t$ and $X_{1}$ and $X_{2}$ are independent, it easily follows that $X(t) \stackrel{\mathfrak{L}}{\rightarrow} X(0)$. To see that $X(t)$ is proper and o.s.s. with exponent $D$ is now trivial. Q.E.D.

We remark that $X_{1}$ above may be chosen to be normally distributed with mean zero and an appropriate covariance operator.

Proof of Corollary 3. We need only prove Corollary 3 in the case that zero is an eigenvalue of the exponent $D$. According to Theorem 3, zero is a simple root of the minimal polynomial $f(x)$ of $D$, so $f(x)=x g(x)$ where $g(0) \neq 0$. Let $\mathscr{w}_{0}=$ kernel $D$ and $\mathscr{\psi} \int_{1}=$ kernel $g(D)$ so that $\mathscr{V}=\mho_{0} \oplus \mathscr{W}$. Let $F_{0}$ denote the projection 
of $\widetilde{V}$ onto $\mho_{0}$ having kernel $\mho_{1}$. Denote by $D_{0}$ the restriction of $D$ to $\mho_{0}$. Similarly let $F_{1}$ be the projection of $\mathscr{V}$ onto $\mathscr{W}_{1}$ having kernel $\mathscr{W}_{0}$ and denote by $D_{1}$ the restriction of $D$ to $\mho_{1}$. Then $F_{0}$ and $F_{1}$ commute with $D$ (this is easy to see since $\mho_{0}$ and $\mho_{1}$ are invariant under $\left.D\right)$ so $\left\{F_{0} X(t): t \geqslant 0\right\}$ and $\left\{F_{1} X(t): t \geqslant 0\right\}$ are o.s.s. and proper in $\mathscr{W}_{0}$ and $\mathscr{W}_{1}$ respectively. Furthermore, $\left\{F_{0} X(t)\right\}$ and $\left\{F_{1} X(t)\right\}$ have exponents $D_{0}$ and $D_{1}$ and centering functions $F_{0} d(r)$ and $F_{1} d(r)$ respectively. Now according to Theorem 1, there is a vector $x_{1}$ in $\mho_{1}$ such that $F_{1} d(r)=\left(I-r^{D_{1}}\right) x_{1}$. On the other hand, $D_{0}=0$, so $F_{0} X(r t) \stackrel{\stackrel{\mathcal{e}}{=}}{=} F_{0} X(t)+F_{0} d(r)$. Let $t \rightarrow 0$ to see that $F_{0} X(0) \stackrel{\mathfrak{e}}{=} F_{0} X(0)+F_{0} d(r)$. This last equality implies that $F_{0} d(r)=0$ for all $r$. But $d(r)=F_{0} d(r)+F_{1} d(r)=\left(I-r^{D_{1}}\right) x_{1}$. Put $x=x_{1}$. Since $x \in \mathcal{W}_{1}, d(r)=$ $\left(I-r^{D}\right) x$. Q.E.D.

6. Proof of Theorem 4. We will use the same notation as in $\S 3$ and as explained immediately before the statement of Theorem 4 . The proof involves three lemmas.

LEMMA 6.1. Let $\{X(t): t \geqslant 0\}$ be a proper o.s.s. process which is continuous in probability. If the distribution of $X(0)$ is full in $V$, then there is a version $\{Y(t): t \geqslant 0\}$ of $\{X(t)\}$ which has constant sample paths. In other words, there is a random vector $U$ taking values in $\mathfrak{V}$ such that for all $t \geqslant 0, Y(t)=U$.

Proof. Assume that the distribution of $X(0)$ is full in $\mathfrak{V}$. We begin by showing that if $D$ is an exponent for $\{X(t)\}$, then every eigenvalue of $D$ has zero real part. Suppose not. Then by Theorem 3, there is an eigenvalue of $D$ and hence of $D^{*}$ which has positive real part. It follows that there is a nonzero vector $y$ in $\mathfrak{V}$ such that $\lim _{t \rightarrow 0} t^{D^{*}} y=0$. For all $t \geqslant 0$, let $\mu_{t}$ denote the distribution of $X(t)$. Since for $r>0$, $X(r t) \stackrel{\mathcal{E}}{=} r^{D} X(t)+d(r)$,

$$
\left|\hat{\mu}_{r t}(x)\right|=\left|\hat{\mu}_{r}\left(t^{D^{*}} x\right)\right|, \quad x \in \mathcal{V} .
$$

Let $c$ be any real number and replace $x$ by $c y$ to obtain

$$
\left|\hat{\mu}_{r t}(c y)\right|=\left|\hat{\mu}_{r}\left(c t^{D^{*}} y\right)\right| \text {. }
$$

Now let $t \rightarrow 0$; since $X(t) \stackrel{P}{\rightarrow} X(0), \mu_{t} \rightarrow \mu_{0}$ and

$$
\left|\hat{\mu}_{0}(c y)\right|=\left|\hat{\mu}_{r}(0)\right|=1 \text {. }
$$

According to Proposition 1, (6.3) implies that $\mu_{0}$ is not full contrary to our hypothesis. Thus every eigenvalue of $D$ must have real part equal to zero. But by Theorem 3, this implies that the eigenvalues of $D$ are simple roots of the minimal polynomial of $D$ and thus $D$ is semisimple. Hence, the closure in GL(V) of $\left\{t^{D}\right.$ : $t>0\}$ is a compact group of linear operators on $\mathfrak{V}$. Thus there is a sequence of positive numbers $r_{n}$ converging to zero such that $\left\{r_{n}^{D}\right\}$ converges to some $A \in \mathrm{GL}(\mathcal{V})$. But $r_{n}^{D} \in G_{r_{n}}$ so by part (a) of Lemma 3.2,

$$
X(T) \stackrel{\stackrel{\mathfrak{f}}{=}}{X}(\underline{0}), \quad T \in[0, \infty)^{k}, k=1,2, \ldots
$$

It follows that $\{X(t): t \geqslant 0\}$ has the same finite-dimensional distributions as does the stochastic process $\{Z(t): t \geqslant 0\}$ defined by the equation $Z(t)=X(0)$ for every 
$t \geqslant 0$. Obviously $\{Z(t)\}$ has constant sample paths. Let $Q$ denote the rationals in $[0, \infty)$ and let

$$
D_{0}=\bigcap_{s \in Q}\left\{f \in \mathscr{V}^{[0, \infty)}: f(s)=f(0)\right\}
$$

Then $P\left[\{X(t)\} \in D_{0}\right]=P\left[\{Z(t)\} \in D_{0}\right]=1$. For $\omega \in\left[\{X(t)\} \in D_{0}\right]$ define $Y(t)(\omega)=X(0)(\omega)$ for every $t \geqslant 0$. If $\omega \notin\left[\{X(t)\} \in D_{0}\right]$, put $Y(t)(\omega)=0$ for every $t \geqslant 0$. Then $\{Y(t)\}$ has constant sample paths. Furthermore, since $P\left[\{X(t)\} \in D_{0}\right]$ $=1$, and since $\{X(t)\}$ is continuous in probability, it follows that

$$
Y(t)=X(t) \text { a.s., } t \geqslant 0 .
$$

That is, $\{Y(t)\}$ is a version of $\{X(t)\}$. Q.E.D.

LEMMA 6.2. Let $\{X(t): t \geqslant 0\}$ be a proper o.s.s. process.

(a) If for some exponent $D$ of $\{X(t)\}$, every eigenvalue of $D$ has real part equal to zero, then the distribution of $X(0)$ is full in $\mathfrak{V}$.

(b) Conversely, if the distribution of $X(0)$ is full in $\mathfrak{V}$, then every eigenvalue of every exponent has zero real part.

Proof. The proof of (b) is contained in the first part of the proof of Lemma 6.1 and so we only need to prove (a). Let $D$ be an exponent for $\{X(t)\}$ and let $d$ : $[0, \infty) \rightarrow \mathcal{V}$ be a map such that for every $r>0\{X(r t): t \geqslant 0\}$ and $\left\{r^{D} X(t)+d(r)\right.$ : $t \geqslant 0\}$ have the same finite-dimensional distributions. Then if $\mu_{t}$ denotes the distribution of $X(t)$,

$$
\mu_{t}=t^{D} \mu_{1} * \delta(d(t)), \quad t>0 .
$$

Now suppose that $\mu_{0}$ is not full. Then there exists a nonzero vector $y$ in $\mathscr{V}$ such that for every real number $c$

$$
\left|\hat{\mu}_{0}(c y)\right|=1 .
$$

Since $\{X(t)\}$ is continuous in law,

$$
\lim _{t \downarrow 0}\left|\hat{\mu}_{1}\left(c t^{D^{*}} y\right)\right|=1 \text {. }
$$

But according to Theorem 3, the eigenvalues of $D$ are simple roots of the minimal polynomial of $D$ and $D^{*}$. It follows that $\left\{t^{D^{*}}: t>0\right\}$ has a compact closure in $\operatorname{GL}(\mathcal{V})$. Hence there exists a sequence $\left\{t_{n}\right\}$ of positive numbers tending to zero such that $\lim _{n \rightarrow \infty} t_{n}^{D^{*}} y$ exists and is not zero. Let $z=\lim _{n \rightarrow \infty} t_{n}^{D^{*}} y$. Then for every real number $c$, we obtain from (6.9)

$$
\left|\hat{\mu}_{1}(c z)\right|=1
$$

which implies that $\mu_{1}$ is not full contrary to our hypothesis. Thus $\mu_{0}$ is full. Q.E.D.

LEMMA 6.3. Let $\{X(t): t \geqslant 0\}$ be proper, continuous in probability and o.s.s. Let $D$ be an exponent for $\{X(t)\}$ and let $\mathcal{V}_{1}$ and $\mathcal{V}_{2}$ be D-invariant subspaces such that $\mathfrak{V}=\mathcal{V}_{1} \oplus \mathcal{V}_{2}$. If $F$ denotes the projection onto $\mathcal{V}_{1}$ with kernel $\mathcal{V}_{2}$, and if $D_{1}$ denotes the restriction of $D$ to $\mathscr{V}_{1}$, then $\{F X(t): t \geqslant 0\}$ is an o.s.s. process which takes values in $\mathcal{V}_{1}$, which is proper in $\mathcal{V}_{1}$, which is continuous in probability, and which has exponent $D_{1}$. 
Proof. First, note that $F$ commutes with $D$. (Indeed, if $x \in \mathfrak{V}$, then $x=x_{1}+x_{2}$ where $x_{1} \in \mathcal{V}_{1}$ and $x_{2} \in \mathscr{V}_{2}$. So $D x_{1} \in \mathscr{V}_{1}$ and $D x_{2} \in \mathscr{V}_{2}$ and hence $F D x=D x_{1}$. On the other hand, $F x=x_{1}$ so $D F x=D x_{1}=F D x$.) It follows that for every $t \geqslant 0, F$ commutes with $t^{D}$. Now according to Theorem 1 , there is a map $d:[0, \infty) \rightarrow \mathcal{V}$ such that for every $r>0\{X(r t): t \geqslant 0\}$ and $\left\{r^{D} X(t)+d(r): t \geqslant 0\right\}$ have the same finite-dimensional distributions. Consequently, $\{F X(r t): t \geqslant 0\}$ and $\left\{r^{D_{1}} F X(t)+\right.$ $F d(r): t \geqslant 0\}$ have the same finite-dimensional distributions. It is now clear that $\{F X(t)\}$ is o.s.s. with exponent $D_{1}$. The continuity in probability of $\{X(t)\}$ implies that of $\{F X(t)\}$ since $F$ is a bounded linear operator. It is easy to see that $\{F X(t)\}$ is proper with respect to $\mathcal{V}_{1}$ since $\{X(t)\}$ is proper. Q.E.D.

Proof of Theorem 4. Let $F_{1}$ denote the projection onto $\mathcal{V}_{1}=\operatorname{kernel} g(D)$ with kernel $\mathscr{V}_{2}$. Then according to Lemma $6.3\left\{F_{1} X(t)\right\}$ is o.s.s. and proper with exponent $D_{1}$ relative to $\Upsilon_{1}$. Since every eigenvalue of $D_{1}$ has real part zero, it follows from Lemma 6.2 that $F_{1} X(0)$ is full in $\mathscr{V}_{1}$. Hence according to Lemma 6.1, there is a version $\left\{X_{1}(t)\right\}$ of $\left\{F_{1} X(t)\right\}$ which has constant sample paths. Now $F_{2}=I-F_{1}$ is the projection of $\mathscr{V}$ onto $\mathscr{V}_{2}$ with kernel $\mathcal{V}_{1}$ so $\left\{X_{2}(t)\right\}=\left\{F_{2} X(t)\right\}$ is o.s.s., continuous in probability, proper with respect to $\mathscr{V}_{2}$, and has exponent $D_{2}=D \mid \mathscr{V}_{2}$. Conclusions (a), (b), and (c) are established and it remains to check (d). Let $\gamma_{t}$ denote the distribution of $X_{2}(t), t \geqslant 0$. Since $X_{2}(t) \stackrel{\mathfrak{L}}{=} t^{D_{2}} X_{2}(1)+d(t)$,

$$
\left|\hat{\gamma}_{t}(y)\right|=\left|\hat{\gamma}_{1}\left(t^{D_{2}^{*}} y\right)\right|, \quad y \in \mathcal{V}_{2} .
$$

Since every eigenvalue of $D_{2}$ has positive real part, it follows that $\lim _{t \rightarrow 0} t^{D_{2}^{*}} y=0$ for all $y \in \mathscr{V}_{2}$. Thus letting $t \rightarrow 0$ in (6.11), we obtain

$$
\left|\hat{\gamma}_{0}(y)\right|=\left|\hat{\gamma}_{1}(0)\right|=1 \text {, }
$$

which implies that $\gamma_{0}$ is degenerate. Q.E.D.

7. Proof of Theorem 5. Let $\{X(t): t \geqslant 0\}$ be a proper stochastic process which is continuous in law. Suppose that there exist a process $\{Y(t)\}$, linear operators $\{A(s)$ : $s>0\}$, and vectors $\{a(s): s>0\}$ such that the finite-dimensional distributions of $\{A(s) Y(s t)+a(s): t \geqslant 0\}$ converge to those of $\{X(t)\}$ as $s \rightarrow \infty$. The proof that $\{X(t)\}$ is o.s.s. will be divided into several lemmas. For each positive integer $k$ and for each $r>0$, define $\mathcal{H}_{r}^{k}$ to be the set of all linear operators $H$ on $\mathcal{V}$ such that for some vector $h \in \mathcal{V}$ and for all $T \in[0, \infty)^{k}$

$$
X(r T) \stackrel{\mathfrak{e}}{=} H X(T)+\underline{h} .
$$

LEMMA 7.1. For all $k$ and $r, \mathcal{H}_{r}^{k}$ is not empty.

Let $r$ and $k$ be arbitrarily chosen and fixed. Then for any $T \in[0, \infty)^{k}$ as $s \rightarrow \infty$

$$
A(s r) Y(s r T)+a(s r) \stackrel{\mathfrak{L}}{\rightarrow} X(T),
$$

and

$$
A(s) Y(s r T)+a(s) \stackrel{\mathfrak{e}}{\rightarrow} X(r T) .
$$


Since $\{X(t)\}$ is proper, it follows that for sufficiently large $s, A(s r)$ and $A(s)$ are invertible. If $\alpha(s) x=A(s) x+a(s)$, then according to Theorem 2.3 of Weissman [15] $\left\{\alpha(n) \alpha(n r)^{-1}\right\}_{n=1}^{\infty}$ is precompact considered as affine transformations on $\mathcal{V}$ (look at the one-dimensional marginals). It follows that they are precompact considered as affine transformations on $\mathcal{V}^{k}$. Thus if $\gamma x=H x+h$ is a limit point, then

$$
X(r T) \stackrel{\mathfrak{R}}{=} H X(T)+\underline{h} \text {. }
$$

Thus $H \in \mathcal{H}_{r}^{k}$. Q.E.D.

LEMMA 7.2. For all $r$ and $k, \mathcal{H}_{r}^{k}$ is closed in the set of linear operators on $\mathfrak{W}$ under the topology induced by the operator norm.

Let $\left\{H_{n}\right\}$ be a sequence in $\mathcal{H}_{r}^{k}$ and suppose that $H_{n} \rightarrow H$. Then for all $T \in[0, \infty)^{k}$ and some vectors $\left\{h_{n}\right\}$ in $\mathcal{V}$

$$
X(r T) \stackrel{\mathfrak{E}}{=} H_{n} X(T)+\underline{h_{n}} .
$$

Since $H_{n} X(T) \stackrel{\mathfrak{L}}{\rightarrow} H X(T)$, it follows that for some $h \in \mathcal{V}, h_{n} \rightarrow h$, and

$$
X(r T) \stackrel{\stackrel{e}{=}}{H} X(T)+\underline{h} .
$$

Thus $H \in \mathcal{H}_{r}^{k}$. Q.E.D.

LEMMA 7.3. For all $r$ and $k, \mathcal{H}_{r}^{k} \supset \mathcal{H}_{r}^{k+1}$.

This is obvious.

LemMa 7.4. For all $r>0, \mathcal{H}_{r}^{1}$ is compact in the set of linear operators on $\mathfrak{V}$.

Since according to Lemma $7.2 \mathcal{K}_{r}^{1}$ is closed, we need only show that $\mathcal{H}_{r}^{1}$ is bounded. Suppose not. Then there is a sequence of linear operators $\left\{H_{n}\right\}$ in $\mathcal{F}_{r}^{1}$ such that $\left\|H_{n}\right\| \rightarrow \infty$. Thus for some sequence $\left\{H_{n}\right\}$ of vectors in $\mathcal{V}$ and for all $t \geqslant 0$

$$
X(r t) \stackrel{\mathfrak{e}}{=} H_{n} X(t)+h_{n}
$$

Then for all $y \in \mathcal{V}$

$$
\left|\hat{\mu}_{r t}(y)\right|=\left|\hat{\mu}_{t}\left(H_{n}^{*} y\right)\right| .
$$

Select unit vectors $y_{n}$ such that $\left\|H_{n}^{*} y_{n}\right\|=\left\|H_{n}^{*}\right\|$. Put $x_{n}=H_{n}^{*} y_{n} /\left\|H_{n}^{*} y_{n}\right\|$. Then $\left\|\left(H_{n}^{*}\right)^{-1} x_{n}\right\| \rightarrow 0$. (Since $\{X(t)\}$ is proper, (7.7) implies that each $H_{n}$ is invertible and hence each $H_{n}^{*}$ is invertible.) Now let $c$ be any real number, and replace $y$ by $c\left(H_{n}^{*}\right)^{-1} x_{n}$ in (7.8) to obtain the equality

$$
\left|\hat{\mu}_{r t}\left(c\left(H_{n}^{*}\right)^{-1} x_{n}\right)\right|=\left|\hat{\mu}_{t}\left(c x_{n}\right)\right| .
$$

Since $\left\|x_{n}\right\|=1$ for all $n$, we may assume $x_{n} \rightarrow$ (some) $x$. Now pass to the limit in (7.9) as $n \rightarrow \infty$ to obtain

$$
\left|\hat{\mu}_{r t}(0)\right|=1=\left|\hat{\mu}_{t}(c x)\right| \text {. }
$$

But (7.10) implies that $\mu_{t}$ is not full contrary to our assumption that $\{X(t)\}$ is proper. Thus, $\mathcal{H}_{r}^{1}$ is bounded and therefore compact. Q.E.D. 
LEMMA 7.5. For all $r>0, \cap_{k=1}^{\infty} \mathcal{H}_{r}^{k}$ is not empty.

Suppose not. Then for some $r, \cap_{k=1}^{\infty} \mathcal{H}_{r}^{k}=\varnothing$ and so by the finite intersection property there exist positive integers $k_{1}<k_{2}<\cdots<k_{j}$ such that

$$
\mathcal{H}_{r}^{k_{1}} \cap \mathcal{H}_{r}^{k_{2}} \cap \cdots \cap \mathcal{H}_{r}^{k_{j}}=\varnothing .
$$

But by Lemma 7.3, $\mathcal{H}_{r}^{k_{j}}=\mathcal{H}_{r}^{k_{1}} \cap \mathcal{H}_{r}^{k_{2}} \cap \cdots \cap \mathcal{H}_{r}^{k_{j}}$, and hence $\mathcal{H}_{r}^{k_{j}}=\varnothing$ which contradicts Lemma 7.1. Thus $\cap_{k=1}^{\infty} \mathcal{F}_{r}^{k} \neq \varnothing$. Q.E.D.

It is now easy to see that $\{X(t)\}$ is o.s.s. Let $r>0$ be arbitrarily chosen and fixed and let $H \in \cap_{k=1}^{\infty} \mathcal{H}_{r}^{k}$. Then for some vector $h_{r}$ and arbitrary $T \in[0, \infty)^{k}$,

$$
X(r T) \stackrel{\mathfrak{e}}{=} H X(T)+\underline{h_{r}} .
$$

This proves that $\{X(t)\}$ is o.s.s.

The converse is trivial. Q.E.D.

8. Proof of Theorems 6 and 7. To prove Theorem 6, let $L$ and $Z$ be as defined just before and in the proof of Lemma 4.3 respectively. First, assume that $\delta(X(t))=\theta$, the full orthogonal group. Let 2 denote the set of all skew-symmetric linear operators on $\mathfrak{T}$, i.e. $Q \in \mathcal{2}$ if and only if $Q+Q^{*}=0$. For $x$ and $y$ in $T(G)$, set $[x, y]=x y-y x$. For real numbers $s$ and $t$ sufficiently small $Z(s x, t y)$ has a power series expansion given by the Campbell-Baker-Hausdorff formula and the coefficient of $s t$ in that expansion is a constant multiple of $[x, y]$. On the other hand,

$$
L(Z(s x, t y))=L(s x)+L(t y)=s L(x)+t L(y),
$$

so $L([x, y])=0$. Thus for all $x$ and $y$ in $T(G),[x, y] \in T(\mathcal{S}(X(t)))=T(\vartheta)=2$.

Now let $D$ be an exponent for $\{X(t)\}$ and let $Q \in \mathcal{2}$. Then $[D, Q] \in \mathcal{2}$ so $[D, Q]+[D, Q]^{*}=0$. That is, $D Q-Q D+\left(-Q D^{*}+D^{*} Q\right)=0$, or $\left(D+D^{*}\right) Q=$ $Q\left(D+D^{*}\right)$.

Thus $D+D^{*}$ commutes with every $Q \in \mathcal{Q}$ and hence with every rotation, since every rotation is of the form $e^{Q}$ for some $Q \in \mathcal{Q}$. (This latter fact is easy to see from p. 274 of Curtice [3].) The only subspaces invariant under all rotations are $\{0\}$ and $\mathscr{T}$, so by Schur's Lemma (Lang [10,p. 173]), $D+D^{*}=c I$ for some number $c$. Define $Q_{0}$ to be (c/2)I-D and note that $Q_{0} \in \mathcal{2}$. By Theorem 2, $(c / 2) I=D+Q_{0}$ is an exponent for $\{X(t)\}$.

Now assume $\delta(X(t))$ is conjugate to $\theta$, i.e. for some positive-definite selfadjoint $W, \delta(X(t))=W \vartheta W^{-1}$. Then $\delta\left(W^{-1} X(t)\right)=\vartheta$. Hence, for some real number $c, c I$ is an exponent for $W^{-1} X(t)$. Thus, $W(c I) W^{-1}$ is an exponent for $\{X(t)\}$. But, $W(c I) W^{-1}=c I$. Q.E.D.

The above proof only required that $\{X(t)\}$ be continuous in law, but the original definition of self-similar requires continuity in probability.

Finally we prove Theorem 7. The assumptions on $\{X(t)\}$ imply that for all $t \geqslant 0$, $\hat{\mu}_{t}(y)=\exp \{t \psi(y)\}$ where $\psi$ arises from the infinitely divisible representation of $\hat{\mu}_{1}$. We first show that if $\{X(t)\}$ is a proper o.s.s. process, then its centering function is identically equal to zero. Since $\mu_{s t}=t^{D} \mu_{s} * \delta(d(t))$, we have $\mu_{0}=t^{D} \mu_{0} * \delta(d(t))$. Since $\mu_{0}=\delta(0), d(t) \equiv 0$. Now assume $\{X(t)\}$ is a proper o.s.s. process and let $\nu$ be the distribution of $X(1)$. Then $\hat{\nu}(y)=\exp \{\psi(y)\}$ and hence $\nu^{t}=\mu_{t}$ for all $t>0$. 
Therefore $\nu^{t}=t^{D} \mu_{1}=t^{D} \nu$, i.e. $X(1)$ is operator-stable with exponent $D$ and centering function identically zero. Conversely, assume $\nu=t^{D} \nu$ for all $t>0$. Hence $\mu_{t}=t^{D} \nu$ and we have $\mu_{s t}=\nu^{s t}=s^{D} t^{D} \nu=s^{D} \mu_{t}$. Q.E.D.

\section{REFERENCES}

1. P. Billingsley, Convergence of types in k-spaces, Z. Wahrsch. Verw. Gebiete 5 (1966), 175-179.

2. P. M. Cohn, Lie groups, Cambridge Tracts in Mathematics and Mathematical Physics, No. 46, Cambridge Univ. Press, 1965. 1974.

3. C. W. Curtice, Linear algebra, An introductory approach, 3rd ed., Allyn and Bacon, Boston, Mass.,

4. V. V. Gorodetskii, On convergence to semi-stable Gaussian processes, Theory Probab. Appl. 22 (1977), 498-508.

5. K. Hoffman and R. Kunze, Linear algebra, 2nd ed., Prentice-Hall, Englewood Cliffs, N. J., 1971.

6. H. Kesten and F. Spitzer, A limit theorem related to a new class of self-similar processes, Z. Wahrsch. Verw. Gebiete 50 (1979), 5-25.

7. R. G. Laha and V. K. Rohatgi, Operator self-similar stochastic processes in $R_{d}$, Stochastic Processes Appl. 12 (1982), 73-84.

8. J. W. Lamperti, Semi-stable stochastic processes, Trans. Amer. Math. Soc. 104 (1962), 62-78.

9. , Semi-stable Markov processes. I, Z. Wahrsch. Verw. Gebiete 22 (1972), 205-225.

10. S. Lang, Analysis. II, Addison-Wesley, Reading, Mass., 1969.

11. M. Sharpe, Operator-stable probability distributions on vector groups, Trans. Amer. Math. Soc. 136 (1969), 51-65.

12. Ya. Sinai, Self-similar probability distributions, Theory Probab. Appl. 21 (1976), 64-80.

13. M. S. Taqqu, A representation for self-similar processes, Stochastic Processes Appl. 7 (1978), 55-64.

14. __ Self-similar processes and related ultraviolet and infrared catastrophes, Proc. Internat. Colloq. Random Fields, June, 1979 (1980) (to appear).

15. I. Weissman, On convergence of types and processes in Euclidean spaces, Z. Wahrsch. Verw. Gebiete 37 (1976), 35-41.

Department of Mathematics, Auburn University, Auburn, Alabama 36849

Department of Mathematics, University of Utah, Salt Lake City, Utah 84112 